\title{
Transient Elastography (Fibroscan) Compared to Diagnostic Endoscopy in the Diagnosis of Varices in Patients with Cirrhosis
}

\author{
Mahmoud Hassan Al Ghamdi ${ }^{1}$, Hind I. Fallatah ${ }^{2, ~ *, ~ H i s h a m ~ O . ~ A k b a r ~}{ }^{2}$ \\ ${ }^{1}$ Department of Surgery, King Abdulaziz University, Jeddah, Saudi Arabia \\ ${ }^{2}$ Hepatology Unit, Department of Internal Medicine, King Abdulaziz University, Jeddah, Saudi Arabia \\ Email address: \\ hindfallatah@hotmail.com (H. I. Fallatah) \\ *Corresponding author
}

\section{To cite this article:}

Mahmoud Hassan Al Ghamdi, Hind I. Fallatah, Hisham O. Akbar. Transient Elastography (Fibroscan) Compared to Diagnostic Endoscopy in the Diagnosis of Varices in Patients with Cirrhosis. Science Journal of Clinical Medicine. Vol. 5, No. 6, 2016, pp. 55-59. doi: $10.11648 /$ j.sjcm.20160506.13

Received: November 26, 2016; Accepted: January 3, 2017; Published: January 24, 2017

\begin{abstract}
We evaluated the effectiveness of the Transient elastography using (Fibroscan) for the prediction of varices and portal hypertensive gastropathy in patients with chronic liver disease. We performed a cross-sectional study of patients with compensated chronic liver disease who had never experienced variceal bleeding and underwent both a Fibroscan assessment for liver fibrosis and a diagnostic UGIE no more than 3 months apart. We collected the patients' demographic data, serum ALT and platelet count values, and Fibroscan and endoscopy results. We included 75 patients in the final analysis; 39 (52\%) had males. Hepatitis $\mathrm{C}$ was the most common diagnosis $(27,36 \%)$. A total of 37 patients $(49.3 \%)$ had either gastric or esophageal varices $(\mathrm{OV})$, portal hypertensive gastropathy (PHG), or both. The mean stiffness score was $30.1 \mathrm{kPa}(\mathrm{SD} 1.2 \mathrm{kPa}$ ). The Fibroscan score was highly correlated with the presence of varices $(\mathrm{r}=.85$ and $\mathrm{P}=.002)$. The mean stiffness score was higher in patients with OVs or PHG than in patients without OVs or PHG, (34.5, SD 18.3 and 25.8, SD 14.9, respectively, P=.027), but no difference was found in the stiffness scores between the patients with small and large varices. The ROC analysis of a stiffness score showed AUC of.67 for the detection of varices. In conclusion: Fibroscan can predict the presence of varices and PHG in patients with cirrhosis, but it cannot distinguish between small and large varices.
\end{abstract}

Keywords: Fibroscan, Esophageal Varices, Cirrhosis, Portal Hypertensive Gastropathy, Endoscopy

\section{Introduction}

Variceal bleeding is one of the leading causes of mortality and morbidity in cirrhotic patients $[1,2]$. The early detection of esophageal varices (OVs) and initiation of primary prophylactic measures will reduce the chance of variceal bleeding and morbidity in patients with cirrhosis [2-4]. Upper gastrointestinal endoscopy is the gold standard method for the detection of OVs [2-4]. However, endoscopy is an invasive method with potential complications, especially in patients with advanced cirrhosis $[5,6]$. Several reports have evaluated the detection of OVs using noninvasive methods to replace the need for invasive endoscopy [7-9]. The transient elastography using (Fibroscan) is currently extensively utilized for the assessment of liver fibrosis, and several reports have shown its value for the prediction of OVs or variceal bleeding $[9,10]$. However, different reports have suggested different cut off values [9-12]. The Baveno VI Consensus suggested that patients with a Fibroscan score of less than $20 \mathrm{kpa}$ and a platelet count of more than 150,000 have a very low risk of varices that require treatment and that screening endoscopy can be deferred [4]. We conducted this study at the King Abdulaziz University Hospital Hepatology Unit to evaluate the accuracy of the Fibroscan tool for the detection of portal hypertensive gastropathy (PHG), OVs and gastric varices. 


\section{Materials and Methods}

\subsection{Study Design}

This was a cross-sectional study.

The study period was from September 2012 to August 2013.

Approval for this study was obtained from the King Abdulaziz University Faculty of Medicine Ethical Committee.

\subsection{Patients}

Patients who had compensated chronic liver disease of any etiology without a previous history of variceal bleeding and had agreed to undergone both a Fibroscan examination and a screening upper gastrointestinal endoscopy not more than 3 months apart during the study period were identified from the hepatology and endoscopy unit database at King Abdulaziz University Hospital.

\subsection{Inclusion Criteria}

We enrolled patients who had a Fibroscan score of 10.3 $\mathrm{kPa}$ or more after 10 successful readings, at least a $75 \%$ success rate and an IQR of $30 \%$ or less according to the manufacturer's recommendations. The value of 10.3 is accepted as the lower cut off value for the detection of previously reported cirrhosis [13-15].

\subsection{Exclusion Criteria}

Patients with incomplete Fibroscan data, lab results or endoscopy data were excluded from the study.

We obtained the demographic data of all the patients (age and sex) from their electronic files. We also collected data on the cause of liver disease and the results of the serum alanine transferase measurement and platelet count. The Fibroscans were performed by two expert hepatologists. We reviewed the hepatology unit database for the results of the Fibroscan, and we obtained the stiffness scores for all patients using the Fibroscan 502 (2005) manufactured by Echosens (Paris, France).

\subsection{Endoscopy}

Endoscopies were performed by two senior expert gastroenterologist hepatologists. We reviewed the endoscopy unit database and the patients' records to obtain the results of the upper GI endoscopies. We categorized the endoscopic findings as no varices, small grade 1 and 2 varices, large grade 3 and 4 varices, PHG, gastric varices and both varices and PHG.

\subsection{Statistical Analysis}

We used IBM SPSS 22 to perform the statistical analysis on the descriptive data. We used correlation analysis to evaluate the relationship between the Fibroscan scores and the presence of OVs. We used Student's T test and one-way ANOVA to compare the mean stiffness scores and different variables. The Chai square test for the relation between the stiffness score and the presence of varices or PHG. We also used the ROC analysis for the stiffness score.

\section{Results}

Patient's characteristics:

During the study period, 75 patients fulfilled the inclusion criteria, and a similar number of males and females were enrolled (39 (52) and 36 (48), respectively). The mean age was 51.34 years (SD 15.2, 19-77 years), with no significant difference between males and females.

The most common cause of liver disease was CHC, Table 1.

Table 1. The distribution of causes of liver disease among the included patients.

\begin{tabular}{lll}
\hline Cause of liver cirrhosis & Number of patients & Percent \\
\hline CHC & 27 & 36.0 \\
CHB & 2 & 2.7 \\
AIH & 12 & 16.0 \\
NAFLD & 15 & 20.0 \\
Bilharzia & 1 & 1.3 \\
Alcohol & 2 & 2.7 \\
Congenital liver fibrosis & 2 & 2.7 \\
PBC & 1 & 1.3 \\
PSC & 1 & 1.3 \\
Wilson's disease & 2 & 2.7 \\
Hemochromatosis & 1 & 1.3 \\
Chronic hepatitis B and C coinfection & 1 & 1.3 \\
Unknown etiology & 8 & 10.7 \\
Total & 75 & 10 \\
\hline
\end{tabular}

CHC (chronic hepatitis C), CHB (chronic hepatitis B), AIH (autoimmune hepatitis), NAFLD (none alcoholic fatty liver disease), PBC (primary biliary cirrhosis), PSC (primary sclerosing cholangitis).

Lab results:

The median serum ALT level was $72 \mathrm{IU} / \mathrm{L}$ (10-350 IU/L), and the median platelet count was $144 \mathrm{~K} / \mu \mathrm{l}(30-435 \mathrm{~K} / \mu \mathrm{l})$. Approximately half of the patients had varices, PHG or both, Table 2.

Table 2. The distribution of patients according to the presence or absence of esophageal varices and portal hypertensive gastropathy.

\begin{tabular}{lll}
\hline & Number of patients & Percent \\
\hline No varices or PHG & 38 & 50.7 \\
Grade 1 and 2 OV & 14 & 18.7 \\
Grade 3 and 4 OV & 8 & 10.7 \\
Gastric varix & 2 & 2.7 \\
PHG & 8 & 10.7 \\
OV and PHG & 5 & 6.7 \\
Total & 75 & 100.0 \\
\hline
\end{tabular}

PHG (portal hypertensive gastropathy, OV (esophageal varices)

A total of 69 patients $(92 \%)$ had a Fibroscan score of 12.5 $\mathrm{kPa}$ or more. The mean stiffness score was $30.1 \mathrm{kPa}$ (SD 1.2 $\mathrm{kPa})$. The Fibroscan score was highly correlated with the presence of varices $(\mathrm{r}=.85$ and $\mathrm{P}=.002)$.

Significant differences were observed between the stiffness scores in patients with and without endoscopic evidence of portal hypertension, with means of 34.5 (SD 18.3 ) and 25.8 (SD 14.9), respectively $(\mathrm{P}=.027)$. No difference in the stiffness score was observed on the post hoc test among the three groups (PHG, small varices and large 
varices), but patients who had both $\mathrm{OV}$ and PHG had stiffness scores significantly higher than those with small varices $(\mathrm{P}=.042)$. We detected OVs and/or PHG in 5 patients $(6.6 \%)$ who had a fibrosis score of less than 13 . However, 7 (9.3\%) patients with a Fibroscan score higher than the mean of patients with varices did not have OVs or PHG. Moreover, no difference was found in the stiffness scores between males and females, and no difference in age was observed between patients with $\mathrm{PHG}$ and/or OVs and those without PHG or OV. No differences were found in the serum ALT level and platelet count between patients with positive endoscopic findings of portal hypertension and those with negative endoscopy. On multiple regression analysis higher stiffness score and younger age, but not platelets count or serum ALT were more likely to be associated with the presence of varices or PHG. Table 3.

Table 3. Multiple regression analysis of relation of presences of esophageal varices or portal hypertensive gastropathy to different to age, platelets count and stiffness score.

\begin{tabular}{|c|c|c|c|c|c|c|}
\hline \multicolumn{7}{|c|}{ Coefficients $^{\mathrm{a}}$} \\
\hline \multirow{2}{*}{ Model } & & \multicolumn{2}{|c|}{ Unstandardized Coefficients } & \multirow{2}{*}{$\begin{array}{l}\text { Standardized Coefficients } \\
\text { Beta }\end{array}$} & \multirow[b]{2}{*}{$\mathbf{t}$} & \multirow[b]{2}{*}{ Sig. } \\
\hline & & B & Std. Error & & & \\
\hline \multirow{6}{*}{1} & (Constant) & .901 & .313 & & 2.873 & .005 \\
\hline & platlets & $1.763 \mathrm{E}-5$ & .001 & .003 & .029 & .977 \\
\hline & ALT & -.001 & .001 & -.093 & -.792 & .431 \\
\hline & age & -.009 & .004 & -.263 & -2.254 & .027 \\
\hline & $\operatorname{sex}$ & -.116 & .113 & -.116 & -1.028 & .308 \\
\hline & stiffness & .008 & .003 & .288 & 2.503 & .015 \\
\hline \multicolumn{7}{|c|}{ a. Dependent Variable: varices or not } \\
\hline
\end{tabular}

Using the Chai square cut off stiffness score of 25Kapa was significantly predicts the presence of OV or PHG P $=.04$ table 4, however applying the Baveno criteria at cut-offs of $20-25 \mathrm{kPa}$ for LS and 150000 for platelet count the would only have diagnose 8 of 37 patients with $\mathrm{OV}$ or PHG with a $P$ value none significant. Another possible reason for that is that in our cohort high mean stiffness score.

Table 4. Cross tabulation of stiffness score above 25 and the presence or absence of varices or portal hypertensive gastropathy.

\begin{tabular}{llll}
\hline & $\begin{array}{l}\text { Stiffness } \\
\text { score above }\end{array}$ & $\begin{array}{l}\text { Stiffness score } \\
\mathbf{2 5} \text { or less }\end{array}$ & total \\
\hline No varices or PHG & 22 & 16 & 38 \\
Detected varices or PHG & 13 & 24 & 37 \\
total & 35 & 40 & 75 \\
\hline
\end{tabular}

PHG (portal hypertensive gastropathy) $\mathrm{P}=.04$

The ROC analysis of a stiffness score showed an area under the curve of.67 for the detection of OV figure 1.

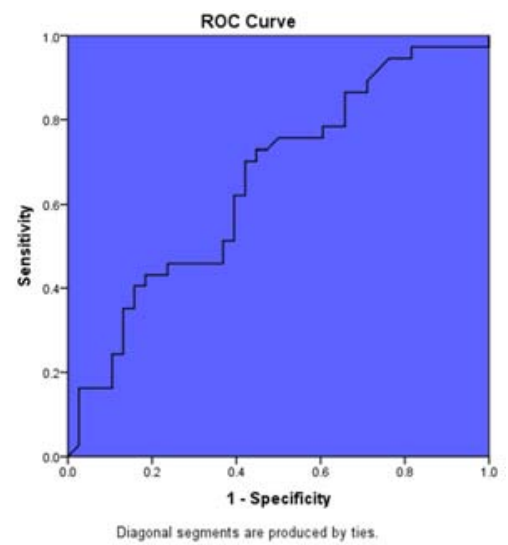

Figure 1. ROC analysis for stiffness score in the detection of varices or portal hypertensive gastropathy.

$\mathrm{ROC}=.67$

\section{Discussion}

Our data showed that the Fibroscan stiffness score correlated with the presence of OVs and PHG, we were able to predict $67 \%$ of OV or PHG cases. However, Fibroscan was not efficient for the differentiation of patients with small varices or PHG from patients with large varices or gastric varices. Although all of our patients had F4 fibrosis, more than half of our patients did not have endoscopic evidence of PGH or OVs. Several investigators in similar previous reports have suggested different cut off Fibroscan scores for the detection of OVs in cirrhotic patients [11, 12, and 15]. Castera et al [15] suggested a cut off value of $21.5 \mathrm{kPa}$ for the prediction of grade 2-3 varices. In a similar report, Saad et al [12] suggested a cut off of $29.7 \mathrm{kPa}$ for the prediction of varices and a cut off of $38.2 \mathrm{kPa}$ for the prediction of large varices. Kazemi et al [11] reported a lower cut off value of 19 for the detection of grade or more 2 varices with a negative predictive value (NPV) of $93 \%$ and a cut off of $13.9 \mathrm{kPa}$ for the detection of all varices. Moreover, Foucher et al [10] reported a cut off score of $27.5 \mathrm{kPa}$ for the detection of varices and an NPV of $90 \%$, but with low sensitivity. Taken together with our findings, these reports show no definite cut off Fibroscan score that can be used to predict OVs and PHG with a great level of confidence. The difference in the cut off Fibroscan scores from different reports can be explained by the variations in the studied cohorts of patients and the presence of different etiologies of liver cirrhosis. Castera et al [13] reviewed different cut off scores for the detection of liver cirrhosis of various etiologies ranging from 10.3 for $\mathrm{CHB}$ to 17.3 for chronic cholestatic liver disease. In addition, in our cohort, $6.6 \%$ of the patients with relatively low Fibroscan scores had OVs, PHG or both. Failing to identify such patients and initiate early prophylaxis would place those patient at risk for morbidity and mortality if they develop variceal bleeding. Hence, the benefit of diagnostic endoscopy outweighs the risk of morbidity and mortality in those patients [2, 3, 4, and 16]. 
Detection of large varices and the initiation of medical prophylaxis are the main reasons that endoscopic screening for OVs is performed $[2-4,16]$. Screening endoscopy to rule out varices in our patients is additionally supported by the relatively high mean Fibroscan score of $25.3 \mathrm{kPa}$ in more than half of our patients who did not have varices or PHG. Taken together with previous work, our findings can be used to defer the need for diagnostic endoscopy in patients with Fibroscan scores lower than the cirrhotic range [4]. However, in the absence of a clearly defined value for varices, it is worthwhile to endoscopically screen all cirrhotic patients to initiate prophylaxis in appropriate patients. Currently the need for medical prophylaxis cannot yet be determined based on Fibroscan findings. Moreover, an additional benefit of endoscopy is the possibility of applying prophylactic band ligation for large varices during the procedure. In addition, endoscopy is essential for the diagnosis of severe forms of PGH or gastric antral ectasia that can cause chronic blood loss and anemia without massive upper gastrointestinal bleeding [17]. In our cohort five patients who had both varices and PHG had higher Fibroscan scores compared to the rest of our patients. This finding might be related to the presence of more severe portal hypertension, but it will require further study with a large number of similar patients to explore this finding.

The use of the Fibroscan examination along with other noninvasive tests or with less invasive abdominal ultrasound examinations for the assessment of liver fibrosis has been shown to be effective in the detection of large varices, but this approach requires more exploration to determine clear cut off Fibroscan and other test values[15, 18, 19]. In our report, the platelet count was not different between the two groups. Castera et al [15] showed that the Fibroscan score and platelet count can be used together to predict the presence of varices. The differences in our results can be explained by our inclusion of only patients with high Fibroscan scores. Calvaruso et al [20] demonstrated that additional splenic stiffness measurements by Fibroscan and the use of noninvasive markers, in particular the platelet count, can predict different sizes of OVs. When we applied the Baveno

Criteria [4] in our cohort most of the patients with varices or PHG were not identified. The reason for that might be that we looked for both varices and PGH in our study while Baveno criteria was aimed to identify patients with varices only. [4]

The cause of liver disease was not identified in $10.7 \%$ of our patients; all these patients had negative viral markers and autoimmune profiles. Most of these patients may have had underlying, long-standing NAFLD that was not diagnosed before cirrhosis developed. This supposition can be supported by the increasing burden of NAFLD in Saudi Arabia [21, 22].

\section{Conclusion}

Our data showed that the Fibroscan score can predict the presence of varices and PHG in cirrhotic patients; however, it was not effective for differentiating small from large varices. In addition, the current suggested cut off values for the Fibroscan score can misdiagnose some patients with OVs.

\section{Author Contributions}

All the three authors contributed in planning the study. Dr Mahmoud worked on the data collection and the first draft of the study. Prof Hisham and Dr Hind contributed in patients recruitments, performing the fibroscan, writing and editing of the paper. Dr Hind contributed in reviewing the final draft.

\section{References}

[1] Fallatah HI, al Nahdi H, al Khatabi M, Akbar HO, Qari YA, Sibiani AR, Bazaraa S. Variceal hemorrhage: Saudi tertiary center experience of clinical presentations, complications and mortality. World J Hepatol 2012; 4: 268-273 [PMID: 23060972 DOI: 10.4254/wjh.v4.i9.268].

[2] Garcia-Tsao G, Sanyal AJ, Grace ND, Carey W. Prevention and management of gastroesophageal varices and variceal hemorrhage in cirrhosis. Hepatology 2007; 46: 922-938 [PMID: 17879356 DOI: 10.1002/hep.21907].

[3] Kim DH, Park JY. Prevention and management of variceal hemorrhage. Int J Hepatol 2013; 2013: 6 [PMID: 23606973 DOI: $10.1155 / 2013 / 434609]$.

[4] de Franchis R. Expanding consensus in portal hypertension: report of the baveno VI consensus workshop: stratifying risk and individualizing care for portal hypertension. $J$ Hepatol 2015; 63: 743-752 [PMID: 26047908 DOI: 10.1016/j.jhep.2015.05.022].

[5] Palmer KR. Complications of gastrointestinal endoscopy. Gut. 2007;56 (4): 456-457. [PMCID: PMC1856860 DOI: 10.1136/gut.2006.105577.

[6] Krystallis C, Masterton GS, Hayes PC, Plevris JN. Update of endoscopy in liver disease: More than just treating varices. WJG. 2012;18 (5): 401-411. [PMCID: PMC3272639 DOI:10.3748/wjg.v18.i5.401.

[7] Cherian JV, Deepak N, Ponnusamy RP, Somasundaram A, Jayanthi V. Non-invasive predictors of esophageal varices. Saudi J Gastroenterol 2011; 17: 64-68 [PMID: 21196656 DOI: 10.4103/1319-3767.74470].

[8] Thomopoulos KC. Non-invasive prediction of esophageal varices: is it possible? Saudi J Gastroenterol 2011; 17: 1-3 [PMID: 21196644 DOI: 10.4103/1319-3767.74426].

[9] Stefanescu H, Grigorescu M, Lupsor M, Maniu A, Crisan D, Procopet B, Feier D, Badea R. A new and simple algorithm for the noninvasive assessment of esophageal varices in cirrhotic patients using serum fibrosis markers and transient elastography. J Gastrointestin Liver Dis 2011; 20: 57-64 [PMID: 21451799].

[10] Foucher J, Chanteloup E, Vergniol J, Castera L, le Bail B, Adhoute X, Bertet J, Couzigou P, de Ledinghen V. Diagnosis of cirrhosis by transient elastography (FibroScan): a prospective study. Gut 2006; 55: 403-408 [PMID: 16020491 DOI: 10.1136/gut.2005.069153].

[11] Kazemi F, Kettaneh A, N'kontchou G, Pinto E, Ganne-Carrie $\mathrm{N}$, Trinchet J-C, Beaugrand M. Liver stiffness measurement selects patients with cirrhosis at risk of bearing large oesophageal varices. J Hepatol 2006; 45: 230-235 [PMID: 16797100 DOI: 10.1016/j.jhep.2006.04.006]. 
[12] Saad Y, Said M, Idris MO, Rabee A, Zakaria S. Liver stiffness measurement by fibroscan predicts the presence and size of esophageal varices in egyptian patients with HCV related liver cirrhosis. J Clin Diagn Res 2013; 7: 2253-2257 [PMID: 24298490 DOI: $10.7860 / \mathrm{jcdr} / 2013 / 6026.3484]$.

[13] Castera L, Forns X, Alberti A. Non-invasive evaluation of liver fibrosis using transient elastography. J Hepatol 2008; 48: 835-847 [PMID: 18334275 DOI: 10.1016/j.jhep.2008.02.008].

[14] Castera L, Vergniol J, Foucher J, le Bail B, Chanteloup E, Haaser M, Darriet M, Couzigou P, de Ledinghen V. Prospective comparison of transient elastography, Fibrotest, APRI, and liver biopsy for the assessment of fibrosis in chronic hepatitis C. Gastroenterology 2005; 128: 343-350 [PMID: 15685546 DOI: 10.1053/j.gastro.2004.11.018].

[15] Castera L, le Bail B, Roudot-Thoraval F, Bernard PH, Foucher J, Merrouche W, Couzigou P, de Ledinghen V. Early detection in routine clinical practice of cirrhosis and oesophageal varices in chronic hepatitis $\mathrm{C}$ : comparison of transient elastography (FibroScan) with standard laboratory tests and non-invasive scores. $J$ Hepatol 2009; 50: 59-68 [PMID: 19013661 DOI: 10.1016/j.jhep.2008.08.018].

[16] Baenas MY, Thalheimer U, Germani G, Burroughs AK. Primary prophylaxis of variceal bleeding. Gastroenterol Hepatol 2011; 7: 560-562 [PMID: 22298996].

[17] Ripoll C, Garcia-Tsao G. Management of gastropathy and gastric vascular ectasia in portal hypertension. Clin Liver Dis 2010; 14: 281-295 [PMID: 20682235 DOI: 10.1016/j.cld.2010.03.013].
[18] Sort P, Muelas M, Isava A, Llao J, Porta F, Puig I, Dominguez-Curell C, Esteve E, Yanguas C, Vida F. Diagnostic accuracy of abdominal ultrasound in the screening of esophageal varices in patients with cirrhosis. Eur $J$ Gastroenterol Hepatol 2014; 26: 1335-1341 [PMID: 25089546 DOI: $10.1097 / \mathrm{meg} .0000000000000174]$.

[19] Bintintan A, Chira RI, Mircea PA. Non-invasive ultrasoundbased diagnosis and staging of esophageal varices in liver cirrhosis. A systematic review of the literature published in the third millenium. Med Ultrason 2013; 15: 116-124 [PMID: 23702501 DOI: 10.11152/mu.2013.2066.152.ab1ric2].

[20] Calvaruso V, Bronte F, Conte E, Simone F, Craxi A, di Marco V. Modified spleen stiffness measurement by transient elastography is associated with presence of large oesophageal varices in patients with compensated hepatitis $\mathrm{C}$ virus cirrhosis. J Viral Hepat 2013; 20: 867-874 [PMID: 24304456 DOI: $10.1111 /$ jvh.12114].

[21] Akbar DH, Kawther AH. Nonalcoholic fatty liver disease in Saudi type 2 diabetic subjects attending a medical outpatient clinic: prevalence and general characteristics. Diabetes Care 2003; 26: 3351-3352 [PMID: 14633828 DOI: 10.2337/diacare.26.12.3351-a].

[22] Al-hamoudi W, El-Sabbah M, Ali S, Altuwaijri M, Bedewi M, Adam M, Alhammad A, Sanai F, Alswat K, Abdo A. Epidemiological, clinical, and biochemical characteristics of Saudi patients with nonalcoholic fatty liver disease: a hospitalbased study. Ann Saudi Med 2012; 32: 288-292 [PMID: 22588441 DOI: $10.5144 / 0256-4947.2012 .288]$. 